\title{
Sperm DNA Fragmentation is Important to Predict ART outcomes
}

\author{
Saeed Zandiyeh* \\ Department of Andrology, Iran \\ *Corresponding author: Saeed Zandiyeh, Department of Andrology, Iran
}

Submission: 韭 August 02, 2018; Published: 海 November 26, 2018

\begin{abstract}
Classical semen and sperm characteristic are poor predictors of outcome of Assisted Reproductive Treatment (ART). Sperm DNA Fragmentation (SDF) index has been generally confirmed as a valuable tool for male fertility evaluation and benefit as a predictor of ICSI and IVF outcome. In this mini-review study set out to the impact of these SDF on male fertility and ART outcomes. The findings of this study suggest that SDF is associated with male infertility and reduce the probability of a successful pregnancy following ART.
\end{abstract}

Keywords: SDF; ART outcome; Pregnancy; Fertilization

\section{Introduction}

Sperm is the male gamete cell which can move and has the ability of capacitation, penetration and fertilization. The aim of sperm is to maintain the generation by transferring the male genetic material into the ovum [1]. The previous studies conducted in humans have demonstrated that it is not always possible to achieve an accurate decision about male fertility according to the results of routine semen examinations [2], and when all the routine sperm parameters are normal, sperm population of an ejaculate may be sub fertile or infertile [3]. Several reports have shown that percentage of DNA damaged sperm in the sperm population of the individuals could affect the fertilization capacity of their sperm, the qualities of their embryos and pregnancy outcomes achieved after ART [4]. Sperm DNA integrity might provide complementary data to predict fertilization capacity of sperm, quality of embryos obtained from these sperms and success rate of ARTs such as IVF and ICSI [1].

\section{DNA Fragmentation in Human Sperm}

It is known that DNA damage occurs in sperm due to various reasons. The most common cause of DNA damage in sperm is oxidative stress. Oxidative stress occurs due to the imbalance between the production of reactive oxygen species and antioxidant defense system [5]. Many different factors can cause oxidative stress and DNA damage in spermatozoa. For example, it was reported that heat stress [6], cryopreservation of sperm [7], and chilling of sperm [8], cause DNA damage in the sperm. It is known that smoking, cancer therapies, varicocele, and cancer can lead to DNA damage in the human sperm [9]. DNA damage can occur in germ cells during spermatogenesis and some DNA damaged germ cells are eliminated via apoptosis [10]. Thus, the disturbances related to the apoptotic mechanism might lead to DNA damaged sperm production. of events where, abnormal cells are marked, and Apoptosis involves activation of endonucleases, which induce DNA strand breaks [11]. Over the past decades, numerous inverse relationships between sperm genetic abnormalities with male infertility and ART outcomes have been reported [11]. In recent years, a number of studies have compared the level of sperm DNA fragmentation between fertile and infertile men. Most studies suggest that sperm DNA fragmentation is more common in infertile patients and may be a contributing factor for their reproductive health. The existing literature associating sperm DNA damage with ART outcomes are controversial some studies show a strong influence of sperm DNA fragmentation on male reproductive health and that sperm DNA testing should be incorporated into routine clinical use [11]. Whereas other clinical reviews do not support the clinical use of sperm DNA fragmentation testing [12]. Previous systematic review studies evaluating Simon et al. [11] observed associating sperm DNA fragmentation with fertilization rate after IVF /ICSI demonstrated that $38 \%(35 / 92)$ of the studies reported a significant inverse relationship between the two parameters. This analysis indicates that a higher proportion of micro drop insemination cases (59\%or19/32) reported a significant inverse relationship between sperm DNA fragmentation and fertilization rate than the ICSI $(24 \%$ or $10 / 42)$ and mixed IVF+ICSI cases $(33 \%$ or $6 / 18)$. They saw that the adverse effect of sperm DNA fragmentation on fertilization rate is more common in micro drop treatment than ICSI treatment. A meta-analysis by Robinson et al. [13] identified 16 articles which included 14ICSI and 11IVF studies. This meta-analysis was performed on 2,969 couples undergoing ART treatment resulting in 1,252 pregnancies and 225 spontaneous pregnancy losses. The results suggested a significant increase in miscarriage in patients with high DNA damage compared with those with low DNA damage 
(Risk risk (RR): 2.16; 95\% CI: 1.54-3.03; P < 0.0001). The impact of sperm DNA fragmentation on pregnancy loss was observed both is raw semen (RR: 1.65 ; 95\% CI: 1.66-2.33; $\mathrm{P}<0.0001$ ) as well as prepared sperm (RR: 3.47 ; 95\% CI: 2.13-5.63; $\mathrm{P}<0.0001$ ) populations [13]. It is notable that previous meta-analysis performed on fewer studies resulted in an adverse effect of sperm DNA fragmentation on spontaneous pregnancy loss.

\section{Conclusion}

The main function of the sperm is to deliver the haploid genetic material to the oocyte. Physiological abnormalities in sperm (in terms of motility and morphology) may prevent the sperm reaching the oocyte there by increasing the time for natural conception, and/or most frequently resulting in male infertility. Abnormalities in the sperm genetic material are common in the sperm of infertile men. Although some studies suggest that a weak association between DNA abnormalities in sperm with ART outcomes, yet a more recent meta- analysis combining all the studies reports a significant association between the two parameters. The evidence presented here associating sperm DNA abnormalities with male infertility and ART outcomes favor the use of these test parameters as a potential and clinically useful biomarker [14]. Supplementation of these tests (DFI) along with classic semen analysis could help a comprehensive diagnosis of men's reproductive health.

\section{References}

1. Kucuk N (2018) Sperm DNA and detection of DNA fragmentations in sperm. Turk J Urol 44(1): 1-5.

2. Samplaski MK, Agarwal A, Sharma R, Sabanegh E (2010) New generation of diagnostic tests for infertility: review of specialized semen tests. Int J Urol 17(10): 839-847.

3. Evenson DP, Larson KL, Jost LK (2002) Sperm chromatin structure assay: its clinical use for detecting sperm DNA fragmentation in male infertility and comparisons with other techniques. J Androl 23(1): 25-43.

4. Sharma RK, Said T, Agarwal A (2004) Sperm DNA damage and its clinical relevance in assessing reproductive outcome. Asian J Androl 6(2): 139148.

5. Dorostghoal M, Kazeminejad SR, Shahbazian N, Pourmehdi M, Jabbari A (2017) Oxidative stress status and sperm DNA fragmentation in fertile and infertile men. Andrologia 49(10): 12762.

6. Banks S, King SA, Irvine DS, Saunders PT (2005) Impact of a mild scrotal heat stress on DNA integrity in murine spermatozoa. Reproduction 129(4): 505-514.

7. Baumber J, Ball BA, Linfor JJ, Meyers SA (2003) Reactive oxygen species and cryopreservation promote DNA fragmentation in equine spermatozoa. J Androl 24(4): 621-628.

8. Hansen BGB, Ersbøll AK, Greve T, Christensen P (2005) Increasing storage time of extended boar semen reduces sperm DNA integrity. Theriogenology 63(7): 2006-2019.

9. Schulte RT, Ohl DA, Sigman M, Smith GD (2010) Sperm DNA damage in male infertility: etiologies, assays, and outcomes. J Assist Reprod Genet 27(1): 3-12.

10. Paul C, Melton DW, Saunders PT (2008) Do heat stress and deficits in DNA repair pathways have a negative impact on male fertility? Mol Hum Reprod 14(1): 1-8.

11. Simon L, Emery BR, Carrell DT (2017) Impact of sperm DNA damage in assisted reproduction. Best Practice \& Research Clinical Obstetrics \& Gynecology.

12.Zini A, Sigman M (2009) Are tests of sperm DNA damage clinically useful? Pros and cons. J Androl 30(3): 219-229.

13. Robinson L, Gallos ID, Conner SJ, Rajkhowa M, Miller D, et al. (2012) The effect of sperm DNA fragmentation on miscarriage rates: a systematic review and meta-analysis. Hum Reprod 27(10): 2908-2917.

14. Palermo GD, Neri QV, Cozzubbo T, Rosenwaks Z (2014) Perspectives on the assessment of human sperm chromatin integrity. Fertil Steril 102(6): $1508-1517$
Creative Commons Attribution 4.0 International License

For possible submissions Click Here
Submit Article

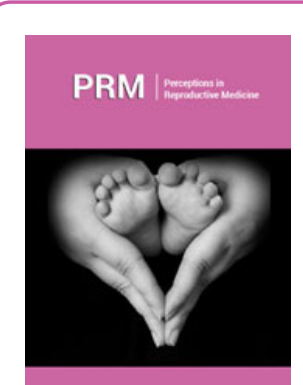

Perceptions in Reproductive Medicine

\section{Benefits of Publishing with us}

- High-level peer review and editorial services

- Freely accessible online immediately upon publication

- Authors retain the copyright to their work

- Licensing it under a Creative Commons license

- Visibility through different online platforms 